\title{
On the joint inversion of SGG and SST data from the GOCE mission
}

\author{
P. Ditmar ${ }^{1}$, P. Visser ${ }^{2}$, and R. Klees ${ }^{1}$ \\ ${ }^{1}$ Physical, Geometrical and Space Geodesy (FMR), Faculty of Civil Engineering and Geosciences (CiTG), Delft University of \\ Technology (TUD), Thijsseweg 11, 2629 JA Delft, The Netherlands \\ ${ }^{2}$ Faculty of Aerospace Engineering, Delft University of Technology, Kluyverweg 1, 2629 HS Delft, The Netherlands
}

\begin{abstract}
The computation of spherical harmonic coefficients of the Earth's gravity field from satellite-to-satellite tracking (SST) data and satellite gravity gradiometry (SGG) data is considered. As long as the functional model related to SST data contains nuisance parameters (e.g. unknown initial state vectors), assembling of the corresponding normal matrix must be supplied with the back-substitution operation, so that the nuisance parameters are excluded from consideration. The traditional back-substitution algorithm, however, may result in large round-off errors. Hence an alternative approach, back-substitution at the level of the design matrix, is implemented. Both a stand-alone inversion of either type of data and a joint inversion of both types are considered. The conclusion drawn is that the joint inversion results in a much better model of the Earth's gravity field than a standalone inversion. Furthermore, two numerical techniques for solving the joint system of normal equations are compared: (i) the Cholesky method based on an explicit computation of the normal matrix, and (ii) the pre-conditioned conjugate gradient method (PCCG), for which an explicit computation of the entire normal matrix is not needed. The comparison shows that the PCCG method is much faster than the Cholesky method.
\end{abstract}

Key words. Earth's gravity field, GOCE, satellite-tosatellite tracking, satellite gravity gradiometry, backsubstitution

\section{Introduction}

GOCE (Gravity Field and Steady-State Ocean Circulation Explorer) is a dedicated gravity field mission to be launched in 2006 within the ESA's Earth Explorer program (ESA, 1999). The aim of the mission is to provide a high-accuracy, high-resolution model of the Earth's static gravity field and of the geoid. The mission will last for 20 months, including two 6-month observation periods. The GOCE orbit will be

Correspondence to: P. Ditmar (ditmar@citg.tudelft.nl) almost circular, with the average altitude between 250 and $270 \mathrm{~km}$. The satellite will be supplied with a sophisticated drag-free control system to compensate for non-gravitational forces like the atmospheric drag and the solar radiation pressure. A GPS receiver on board will provide the data needed for a precise satellite orbit determination. Two measurement techniques will be exploited for gravity field determination: (1) high-low satellite-to-satellite tracking (SST) and (2) satellite gravity gradiometry (SGG). Gravity field determination from high-low SST is not new. This technique is used, for instance, by the ongoing CHAMP mission. Unfortunately, the resolution of Earth's gravity field models that can be derived from high-low SST data is very limited. In order to achieve a higher resolution, SGG data have to be taken into account. The SGG data alone, however, cannot be used for an accurate determination of the long wavelength features in the Earth's gravity field, because the anticipated accuracy of SGG data at low frequencies is poor. Therefore, SST and SGG are complementary, and only a joint inversion of both data sets may provide a sufficiently accurate gravity field model. The paper describes the prototype of an algorithm for the joint inversion of SGG and SST data. Numerical simulations demonstrate that the errors in the models obtained by a stand-alone inversion of SST or SGG data are much larger than the scientific mission objectives allow for. Dramatic improvements can be achieved if both data sets are processed jointly.

\section{Theory}

2.1 Relationships between the collected data and the Earth's gravity field

\subsubsection{SGG data}

SGG data are measurements of the second derivatives of the gravitational potential (i.e. the SGG tensor). Four components of the SGG tensor will be recorded with high accuracy for the purpose of further processing: $\mathrm{XX}, \mathrm{YY}, \mathrm{ZZ}$, and 
$\mathrm{XZ}$ (the $\mathrm{X}$-axis is nominally directed along the track, the $\mathrm{Y}$ axis across the track, and the $\mathrm{Z}$-axis upwards, complementing the frame to a right-handed one). In the course of the pre-processing, the contribution of a reference gravity field is subtracted from the collected data. The remaining signal can be linearly related to the parameters describing the disturbing potential:

$\mathbf{d}_{\text {sgg }}=\mathbf{A}_{\mathcal{G}} \mathbf{x}_{\mathcal{G}}$,

where $\mathbf{d}_{\mathbf{s g g}}$ is the vector of pre-processed SGG data; $\mathbf{A}_{\mathcal{G}}$ is the design matrix which depends on the model parameterization, and $\mathbf{x}_{\mathcal{G}}$ is the vector of parameters describing the disturbing potential. In what follows, we will assume that the disturbing potential is represented as a series of spherical harmonics, so that elements of the vector $\mathbf{x}_{\mathcal{G}}$ are corrections to be applied to the harmonic coefficients of the reference potential.

\subsubsection{SST data}

In the approach adopted, a two-step procedure to handle SST measurements is used. In the first step, a precise orbit determination procedure is exploited, which results in a series of satellite positions. The goal of the second step is to convert the positions into parameters of the Earth's gravity field. The problem is that the gravity field manifests itself not in satellite positions directly but in satellite accelerations, i.e. in second derivatives of positions. There are different ways to relate the satellite positions to the Earth's gravity: to derive accelerations by the double numerical differentiation of positions (Schäfer, 2001) or to make use of the energy conservation low (Wolff, 1969; Jekeli, 1999); the latter approach may be combined with the fast spherical collocation technique (Tscherning, 2002). However, the most common (and mature) way is the orbit integration approach. It starts from the computation of the satellite orbit corresponding to a reference gravity field, which can be done by the numerical integration of the equations of motion. In doing so, one has to specify the initial state of the satellite (satellite position and velocity at the initial moment). A least-square adjustment can be used to find the initial state optimally. As soon as the reference orbit is determined, one should know how it is influenced by variations of the gravity field. This information is contained in the SST-related design matrix, entries of which can be obtained by integrating the variational equations. Importantly, a computation of the gravity field parameters should be accompanied by a new computation of the initial state vector because the optimal estimation of the latter is not independent from the gravity field model. Then, a relationship between the orbit discrepancies and the unknown parameters can be written as a system of linear equations:

$\mathbf{d}_{\text {sst }}=\mathbf{A}_{\mathcal{S}} \mathbf{x}_{\mathcal{G}}+\mathbf{A}_{\mathcal{A}} \mathbf{x}_{\mathcal{A}}$,

where $\mathbf{d}_{\text {sst }}$ is the vector of orbit discrepancies; $\mathbf{x}_{\mathcal{A}}$ is the vector of parameters describing the initial state vector; $\mathbf{A}_{\mathcal{S}}$ is the design matrix part which is related to the gravity field, and $\mathbf{A}_{\mathcal{A}}$ is the design matrix part which is related to the initial state vector.
Strictly speaking, the linear relationship (2) holds only when the reference model is of high quality, so that orbit discrepancies are sufficiently small, otherwise an iterative improvement of the solution may be needed. For the time being, however, we leave this aspect beyond the consideration.

By merging relationships (1) and (2), we can write a joint system of linear equations:

$\mathbf{A x}=\mathbf{d}$

with

$\mathbf{d}=\left(\begin{array}{c}\mathbf{d}_{\text {sgg }} \\ \mathbf{d}_{\text {sst }}\end{array}\right), \quad \mathbf{A}=\left(\begin{array}{cc}\mathbf{A}_{\mathcal{G}} & 0 \\ \mathbf{A}_{\mathcal{S}} & \mathbf{A}_{\mathcal{A}}\end{array}\right), \quad \mathbf{x}=\left(\begin{array}{l}\mathbf{x}_{\mathcal{G}} \\ \mathbf{x}_{\mathcal{A}}\end{array}\right)$.

\subsection{Normal equations}

From the relationships (3) and (4) it follows that the leastsquare estimation of the vector $\mathbf{x}_{\mathcal{G}}$, which describes the disturbing potential, can be found by solving the system of normal equations:

$\mathbf{N} \mathbf{x}_{\mathcal{G}}=\mathbf{y}$.

The right-hand side vector $\mathbf{y}$ is composed of two terms (the SGG-related and the SST-related), whereas the normal matrix $\mathbf{N}$ composed of 3 terms (the SGG-related, the SSTrelated, and the regularization-related):

$\mathbf{N}=\mathbf{N}_{\mathrm{sgg}}+\mathbf{N}_{\mathrm{sst}}+\alpha \mathbf{R}, \quad \mathbf{y}=\mathbf{y}_{\mathrm{sgg}}+\mathbf{y}_{\mathrm{sst}}$

with $\alpha$ standing for the regularization parameter and $\mathbf{R}$ for the regularization matrix. The explicit expressions for SGGrelated terms are straightforward:

$\mathbf{N}_{\text {sgg }}=\mathbf{A}_{\mathcal{G}}{ }^{T} \mathbf{C}_{\text {sgg }}{ }^{-1} \mathbf{A}_{\mathcal{G}}, \quad \mathbf{y}_{\text {sgg }}=\mathbf{A}_{\mathcal{G}}{ }^{T} \mathbf{C}_{\text {sgg }}{ }^{-1} \mathbf{d}_{\text {sgg }}$

with $\mathbf{C}_{\text {sgg }}$ being the covariance matrix of SGG data.

The explicit expressions for SST-related terms can be obtained after elimination of the unknowns related to the initial state vector by means of the back-substitution:

$$
\begin{aligned}
& \mathbf{N}_{\text {sst }}= \\
& \mathbf{A}_{\mathcal{S}}{ }^{T} \mathbf{C}_{\text {sst }}{ }^{-1} \mathbf{A}_{\mathcal{S}}-\mathbf{A}_{\mathcal{S}}{ }^{T} \mathbf{C}_{\text {sst }}{ }^{-1} \mathbf{A}_{\mathcal{A}} \mathbf{N}_{\mathcal{A} \mathcal{A}}{ }^{-1} \mathbf{A}_{\mathcal{A}}{ }^{T} \mathbf{C}_{\text {sst }}{ }^{-1} \mathbf{A}_{\mathcal{S}} \\
& \mathbf{y}_{\text {sst }}= \\
& \mathbf{A}_{\mathcal{S}}{ }^{T} \mathbf{C}_{\text {sst }}{ }^{-1} \mathbf{d}_{\text {sst }}-\mathbf{A}_{\mathcal{S}}{ }^{T} \mathbf{C}_{\mathrm{sst}}{ }^{-1} \mathbf{A}_{\mathcal{A}} \mathbf{N}_{\mathcal{A} \mathcal{A}}{ }^{-1} \mathbf{A}_{\mathcal{A}}{ }^{T} \mathbf{C}_{\text {sst }}{ }^{-1} \mathbf{d}_{\text {sst }}
\end{aligned}
$$

with $\mathbf{C}_{\text {sst }}$ denoting the covariance matrix of SST data and $\mathbf{N}_{\mathcal{A} \mathcal{A}}=\mathbf{A}_{\mathcal{A}}{ }^{T} \mathbf{C}_{\mathrm{sst}}{ }^{-1} \mathbf{A}_{\mathcal{A}}$.

In fact, computations of both the SGG- and SST-related normal matrices according to expressions (7) and (8) suffer from drawbacks: the expression (8) leads to large round-off errors whereas computations prescribed by the formula (7) are simply too time-consuming. More practical algorithms to compute the normal matrices are discussed in the next section. 


\subsection{Computation of the SGG-related normal matrix}

An explicit implementation of Eq. (7) may result in rather time-consuming computations. Let us forget for the moment about the matrix $\mathbf{C}_{\mathbf{s g g}}{ }^{-1}$ (in other words, assume that the noise in the SGG data is white). Assume further that the SGG-related design matrix $\mathbf{A}_{\mathcal{G}}$ is known. As can be easily seen, the number of operations needed to compute the normal matrix according to Eq. (7) is of the order $O\left(N \times M^{2}\right)$, where $N$ is the number of data and $M$ is the number of unknown parameters.

In practice, it is anticipated that noise in SGG data will be colored (i.e. frequency-dependent). Then, the covariance matrix $\mathbf{C}_{\text {sgg }}$ is non-diagonal, so that its inversion can make the computation of the normal matrix even more tedious. Fortunately, it is known that if the noise is stationary, the covariance matrix is Toeplitz. The application of the inverse of such a matrix to a vector can be closely approximated by simply filtering this vector (Schuh, 1996; Klees et al., 2003). For computation of the normal matrix it means that each column of the design matrix should be subject to filtering. Furthermore, it is usually possible to keep the filter length $o$ modest $(o \ll M)$. Then, we can assert that filtering takes not more that $O(N)$ operations per column. Thus, our statement that the number of operations to compute the normal matrix is of the order $O\left(N \times M^{2}\right)$ remains valid in case of the colored noise as well.

In order to reduce the number of operations, we propose to exploit fast synthesis and co-synthesis techniques (Ditmar and Klees, 2002; Ditmar et al., 2003). With synthesis and co-synthesis, we mean the application of the design matrix and of the transposed design matrix, respectively, to a vector. In order to apply these procedures for the computation of the normal matrix, a unit vector $\mathbf{e}_{\mathbf{m}}$ of length $M$ has to be defined. All the elements of this vector are equal to 0 except for the element at the position $m$ : this element is equal to 1 . Sequential application of the synthesis, filtering, and co-synthesis to this vector results in the $m$-th column of the normal matrix:

$\mathbf{N}_{\text {sgg }}{ }^{(m)}=\mathbf{A}_{\mathcal{G}}{ }^{T}\left(\mathbf{C}_{\mathbf{s g g}}{ }^{-1}\left(\mathbf{A}_{\mathcal{G}} \mathbf{e}_{\mathbf{m}}\right)\right)$

The fast synthesis/co-synthesis consists of the initialization/finalization, which requires $O\left(M^{2}\right)$ operations, and the main loop, which needs $O(N)$ operations (Ditmar et al., 2003). For long data sets, time expenditures for the initialization/finalization are minor, so we can state that all three steps - synthesis, filtering, and co-synthesis - require $O(N)$ operations. Then, the total number of operations for the computation of the normal matrix becomes $O(N \times M)$, which results in a dramatic speed-up of the computations. It should be pointed out that the fast co-synthesis may also be used to accelerate the computation of the right-hand side vector $\mathbf{y}_{\mathbf{s g g}}$.

\subsection{Computation of the SST-related normal matrix}

Unlike the case of SGG data, we did not develop an algorithm for computing the SST-related design matrix our- selves. Instead, we import the design matrix from computer files, which are created by the software package GEODYN (Rowlands et al., 1995). The same software is also used to compute orbit discrepancies. Still, we programmed the computation of the SST-related normal matrix. In principle, the equations (8) could be used for that purpose directly because computations in this case are not so time-consuming as those in case of SGG data. This is because the purpose of SST data is to determine only the low-frequency part of the spatial gravity spectrum; hence a much lower sampling rate can be used, so that the total number of SST data is much less than that of SGG data. There is, however, another problem, which is caused by the fact that the numerical integration introduces similarities in columns of matrices $\mathbf{A}_{\mathcal{S}}$ and $\mathbf{A}_{\mathcal{A}}$. As a consequence, the traditional back-substitution prescribed by Eq. (8) results in a subtraction of two very close matrices, which cause significant round-off errors. The influence of the round-off errors is especially strong when the SST-data are inverted in the stand-alone mode (without SGG data). In that case, the computed normal matrix may even loose its positive-definiteness, so that traditional ways to solve the system of normal equations like the Cholesky decomposition become impossible.

There is a number of possible solutions to the problem, e.g. to make computations with enhanced (16-byte) precision. We proposed, however, another approach, which is in our opinion the most straightforward: to carry out the backsubstitution not at the level of the normal matrix but at the level of the design matrix. First of all, assume that the column $m$ of the matrix $\mathbf{A}_{\mathcal{S}}$ has to be approximated by columns of matrix $\mathbf{A}_{\mathcal{A}}$. Assume further that difference between the $m$-the column of the matrix $\mathbf{A}_{\mathcal{S}}$ and a linear combination of columns of the matrix $\mathbf{A}_{\mathcal{A}}$ should be minimized in the sense of the L2-norm with weights $\mathbf{C}_{\text {sst }}{ }^{-1}$ :

$$
\left\|\mathbf{A}_{\mathcal{A}} \boldsymbol{\beta}_{\boldsymbol{m}}-\mathbf{A}_{\mathcal{S}}{ }^{(m)}\right\|_{\mathbf{C}_{\mathbf{s s t}}{ }^{-1}}=\min ,
$$

where vector $\beta_{\mathbf{m}}$ consists of unknown coefficients in the linear combination. Obviously, the minimum of the expression (10) is reached when

$\boldsymbol{\beta}_{\boldsymbol{m}}=\mathbf{N}_{\mathcal{A} \mathcal{A}}{ }^{-1} \mathbf{A}_{\mathcal{A}}{ }^{T} \mathbf{C}_{\mathbf{s s t}}{ }^{-1} \mathbf{A}_{\mathcal{S}}{ }^{(m)}$.

Let us introduce now an updated design matrix $\hat{\mathbf{A}}_{\mathcal{S}}$, which is obtained by subtracting the found approximation from each column of the original design matrix:

$\hat{\mathbf{A}}_{\mathcal{S}}=\mathbf{A}_{\mathcal{S}}-\mathbf{A}_{\mathcal{A}} \mathbf{N}_{\mathcal{A} \mathcal{A}}{ }^{-1} \mathbf{A}_{\mathcal{A}}{ }^{T} \mathbf{C}_{\mathbf{s s t}}{ }^{-1} \mathbf{A}_{\mathcal{S}}$

One can easily see that utilization of the matrix $\hat{\mathbf{A}}_{\mathcal{S}}$ for building the normal matrix results directly in the desirable matrix $\mathbf{N}_{\text {sst }}$ :

$\hat{\mathbf{A}}_{\mathcal{S}}^{T} \mathbf{C}_{\mathbf{s s t}}{ }^{-1} \hat{\mathbf{A}}_{\mathcal{S}}=$

$\mathbf{A}_{\mathcal{S}}{ }^{T} \mathbf{C}_{\mathbf{s s t}}{ }^{-1} \mathbf{A}_{\mathcal{S}}-2 \mathbf{A}_{\mathcal{S}}{ }^{T} \mathbf{C}_{\mathrm{sst}}{ }^{-1} \mathbf{A}_{\mathcal{A}} \mathbf{N}_{\mathcal{A} \mathcal{A}}{ }^{-1} \mathbf{A}_{\mathcal{A}}{ }^{T} \mathbf{C}_{\mathrm{sst}}{ }^{-1} \mathbf{A}_{\mathcal{S}}+$

$\mathbf{A}_{\mathcal{S}}{ }^{T} \mathbf{C}_{\text {sst }}{ }^{-1} \mathbf{A}_{\mathcal{A}} \mathbf{N}_{\mathcal{A} \mathcal{A}}{ }^{-1} \mathbf{A}_{\mathcal{A}}{ }^{T} \mathbf{C}_{\text {sst }}{ }^{-1} \mathbf{A}_{\mathcal{A}} \mathbf{N}_{\mathcal{A} \mathcal{A}}{ }^{-1} \mathbf{A}_{\mathcal{A}}{ }^{T} \mathbf{C}_{\text {sst }}{ }^{-1} \mathbf{A}_{\mathcal{S}}=$ $\mathbf{A}_{\mathcal{S}}{ }^{T} \mathbf{C}_{\text {sst }}{ }^{-1} \mathbf{A}_{\mathcal{S}}-\mathbf{A}_{\mathcal{S}}{ }^{T} \mathbf{C}_{\text {sst }}{ }^{-1} \mathbf{A}_{\mathcal{A}} \mathbf{N}_{\mathcal{A} \mathcal{A}}{ }^{-1} \mathbf{A}_{\mathcal{A}}{ }^{T} \mathbf{C}_{\text {sst }}{ }^{-1} \mathbf{A}_{\mathcal{S}}=\mathbf{N}_{\text {sst }}$; 
in the derivation, we used the equality $\mathbf{N}_{\mathcal{A} \mathcal{A}}{ }^{-1} \mathbf{A}_{\mathcal{A}}{ }^{T} \mathbf{C}_{\text {sst }}{ }^{-1} \mathbf{A}_{\mathcal{A}}=\mathbf{N}_{\mathcal{A} \mathcal{A}}{ }^{-1} \mathbf{N}_{\mathcal{A} \mathcal{A}}=\mathbf{I}$.

The same updated design matrix $\hat{\mathbf{A}}_{\mathcal{S}}$ can be directly used for the computation of the right-hand side vector $\mathbf{y}_{\text {sst }}$ :

$$
\begin{aligned}
& \hat{\mathbf{A}}_{\mathcal{S}}^{T} \mathbf{C}_{\text {sst }}{ }^{-1} \mathbf{d}_{\text {sst }}= \\
& \mathbf{A}_{\mathcal{S}}{ }^{T} \mathbf{C}_{\text {sst }}{ }^{-1} \mathbf{d}_{\text {sst }}-\mathbf{A}_{\mathcal{S}}{ }^{T} \mathbf{C}_{\text {sst }}{ }^{-1} \mathbf{A}_{\mathcal{A}} \mathbf{N}_{\mathcal{A} \mathcal{A}}{ }^{-1} \mathbf{A}_{\mathcal{A}}{ }^{T} \mathbf{C}_{\text {sst }}{ }^{-1} \mathbf{d}_{\text {sst }} \\
& \quad=\mathbf{y}_{\text {sst }} \text {. }
\end{aligned}
$$

Thus, the expression (12) describes the back-substitution at the level of the design matrix.

There is a simple geometrical interpretation of the proposed approach. Let us introduce two projecting operators: (1) operator $\Pi_{\mathcal{R}\left(\mathcal{A}_{\mathcal{A}}\right)}^{C_{s s t}^{-1}}$ for projecting onto the space $\mathcal{R}\left(\mathcal{A}_{\mathcal{A}}\right)$ (the range space of matrix $A_{\mathcal{A}}$ ) with respect to metric $C_{s s t}^{-1}$ :

$\Pi_{\mathcal{R}\left(\mathcal{A}_{\mathcal{A}}\right)}^{C_{s t}^{-1}}=\mathbf{A}_{\mathcal{A}} \mathbf{N}_{\mathcal{A} \mathcal{A}}{ }^{-1} \mathbf{A}_{\mathcal{A}}{ }^{T} \mathbf{C}_{\text {sst }}{ }^{-1}$

and (2) operator $\Pi_{\mathcal{R}^{\perp}\left(\mathcal{A}_{\mathcal{A}}\right)}^{C_{s s}^{-1}}$ for projecting onto the space $\mathcal{R}^{\perp}\left(\mathcal{A}_{\mathcal{A}}\right)$ (the orthogonal space with respect to metric $C_{\text {sst }}^{-1}$ ):

$\Pi_{\mathcal{R}^{\perp}\left(\mathcal{A}_{\mathcal{A}}\right)}^{C_{s t}^{-1}}=\mathbf{I}-\Pi_{\mathcal{R}\left(\mathcal{A}_{\mathcal{A}}\right)}^{C_{s s t}^{-1}}=\mathbf{I}-\mathbf{A}_{\mathcal{A}} \mathbf{N}_{\mathcal{A} \mathcal{A}}{ }^{-1} \mathbf{A}_{\mathcal{A}}{ }^{T} \mathbf{C}_{\mathrm{sst}}{ }^{-1}$.

Application of these operators to matrix $\mathbf{A}_{\mathcal{S}}$ allows one to represented it as sum of two components: the component $\mathbf{A}_{\mathcal{S}}^{\|} \equiv \Pi_{\mathcal{R}\left(\mathcal{A}_{\mathcal{A}}\right)}^{C_{s t}^{-1}} \mathbf{A}_{\mathcal{S}}$ that belongs to the range space $R\left(A_{\mathcal{A}}\right)$ and the perpendicular component $\mathbf{A}_{\mathcal{S}}^{\perp} \equiv \Pi_{\mathcal{R}^{\perp}\left(\mathcal{A}_{\mathcal{A}}\right)}^{C_{S t}^{-1}} \mathbf{A}_{\mathcal{S}}=$ $\hat{\mathbf{A}}_{\mathcal{S}}$. A similar decomposition can be applied to the data vector $\mathbf{d}_{\text {sst }}: \mathbf{d}_{\mathrm{sst}}=\mathbf{d}_{\mathrm{sst}}^{\|}+\mathbf{d}_{\mathrm{sst}}^{\perp}$, where $\mathbf{d}_{\mathrm{sst}}^{\|} \equiv \Pi_{\mathcal{R}\left(\mathcal{A}_{\mathcal{A}}\right)}^{C_{s t}^{-1}} \mathbf{d}_{\mathrm{sst}}$ and $\mathbf{d}_{\text {sst }}^{\perp} \equiv \Pi_{\mathcal{R}^{\perp}\left(\mathcal{A}_{\mathcal{A}}\right)}^{C_{\text {s. }}^{-1}} \mathbf{d}_{\text {sst }}$. Now, the expressions (8) for the SSTrelated normal matrix and the corresponding right-hand side vector can be written as

$\mathbf{N}_{\text {sst }}=\mathbf{A}_{\mathcal{S}}{ }^{T} \mathbf{C}_{\text {sst }}{ }^{-1} \mathbf{A}_{\mathcal{S}}-\mathbf{A}_{\mathcal{S}}{ }^{T} \mathbf{C}_{\text {sst }}{ }^{-1} \mathbf{A}_{\mathcal{S}}^{\|}=\mathbf{A}_{\mathcal{S}}{ }^{T} \mathbf{C}_{\text {sst }}{ }^{-1} \mathbf{A}_{\mathcal{S}}^{\perp}$

$\mathbf{y}_{\text {sst }}=\mathbf{A}_{\mathcal{S}}{ }^{T} \mathbf{C}_{\mathrm{sst}}{ }^{-1} \mathbf{d}_{\mathrm{sst}}-\mathbf{A}_{\mathcal{S}}{ }^{T} \mathbf{C}_{\mathrm{sst}}{ }^{-1} \mathbf{d}_{\mathrm{sst}}^{\|}=\mathbf{A}_{\mathcal{S}}{ }^{T} \mathbf{C}_{\mathrm{sst}}{ }^{-1} \mathbf{d}_{\mathrm{sst}}^{\perp} \cdot{ }^{\perp}$ (17)

The idea of the back-substitution at the level of the design matrix is to replace in these equations the full matrix $\mathbf{A}_{\mathcal{S}}{ }^{T}$ with its projection onto the space $R^{\perp}\left(A_{\mathcal{A}}\right)$ :

$\mathbf{N}_{\text {sst }}=\left(\mathbf{A}_{\mathcal{S}}^{\perp}\right)^{T} \mathbf{C}_{\text {sst }}{ }^{-1} \mathbf{A}_{\mathcal{S}}^{\perp}$

$\mathbf{y}_{\text {sst }}=\left(\mathbf{A}_{\mathcal{S}}^{\perp}\right)^{T} \mathbf{C}_{\text {sst }}{ }^{-1} \mathbf{d}_{\text {sst }}^{\perp}$.

This can be done with impunity because, by definition,

$\left(\mathbf{A}_{\mathcal{S}}^{\|}\right)^{T} \mathbf{C}_{\mathbf{s s t}}{ }^{-1} \mathbf{A}_{\mathcal{S}}^{\perp}=0$

$\left(\mathbf{A}_{\mathcal{S}}^{\|}\right)^{T} \mathbf{C}_{\mathrm{sst}}{ }^{-1} \mathbf{d}_{\mathrm{sst}}^{\perp}=0$.

On the other hand, in practical computations the equalities (19) would not hold exactly due to a limited machine accuracy. As long as elements of the matrix $\mathbf{A}_{\mathcal{S}}^{\|}$are orders of magnitude larger than elements of the matrix $\mathbf{A}_{\mathcal{S}}^{\perp}$, such "minor" errors may noticeably influence the computations if the traditional back-substitution is followed. Back-substitution at the level of the design matrix allows one to avoid the loss of accuracy.

It is worth adding that the noise in SST data has been assumed so far in our studies to be white, so that $\mathbf{C}_{\text {sst }}=\sigma_{s s t}^{2} \mathbf{I}$, where $\sigma_{s s t}$ is the accuracy of SST-data.

2.5 Solving the system of normal equations by means of the conjugate gradient method

We have also implemented an alternative approach to solve the system of normal equations: the method of conjugate gradients with pre-conditioning (PCCG) (Hestenes and Stiefel, 1952; Bertsekas, 1982). At each iteration of this method, one should apply the normal matrix $\mathbf{N}$ to a certain vector $\mathbf{q}$. Importantly, this operation can be done without an explicit computation of the normal matrix. Instead, a sequence of matrix-to-vector multiplication can be carried out as follows:

$\mathbf{N q}=\mathbf{A}_{\mathcal{G}}{ }^{T}\left(\mathbf{C}_{\mathrm{sgg}}{ }^{-1}\left(\mathbf{A}_{\mathcal{G}} \mathbf{q}\right)\right)+\hat{\mathbf{A}}_{\mathcal{S}}^{T}\left(\mathbf{C}_{\text {sst }}{ }^{-1}\left(\hat{\mathbf{A}}_{\mathcal{S}} \mathbf{q}\right)\right)+\alpha \mathbf{R q} \cdot(20)$

The most time-consuming operations here are multiplications of the design matrix $\mathbf{A}_{\mathcal{G}}$ and of the transposed design matrix $\mathbf{A}_{\mathcal{G}}{ }^{T}$ to vectors. The number of PCCG iterations is, however, usually much smaller than the number of unknowns. Hence can expect that the PCCG method would work anyway faster than any method based on the explicit computation of the full normal matrix.

Importantly, a fast convergence of the PCCG method can only be achieved when a suitable pre-conditioner (i.e. a good approximation to the normal matrix) is used. At each PCCG iteration, a system of linear equations has to be solved with the pre-conditioner as the system matrix. In our algorithm, the pre-conditioner $\mathbf{N}^{(\mathbf{p c})}$ is built similarly to the normal matrix itself:

$\mathbf{N}^{(\mathbf{p c})}=\mathbf{N}_{\mathrm{sgg}}^{(\mathbf{p c})}+\mathbf{N}_{\mathrm{sst}}+\alpha \mathbf{R}$.

One can see from Eq. (21) that the computation of the preconditioner differs from that of the true normal matrix only in the part related to the SGG data. More specifically, the matrix $\mathbf{N}_{\text {sgg }}^{(\mathbf{p c})}$ is computed analytically, as a block-diagonal approximation of the SGG-related normal matrix (Colombo, 1986; Ditmar and Klees, 2002). Thus, the time-consuming computation of the true SGG-related normal matrix is avoided.

\section{Computational experiments}

\subsection{Stand-alone inversion of SST data}

The first goal of our simulations was to reveal typical inaccuracies in recovering the Earth's gravity field model from the SST data alone. A set of satellite positions was generated with the GEODYN software according to the following scenario:

- Model of the Earth's gravity field used to compute the "true" orbit: EGM96 (Lemoine et al., 1998) truncated at degree and order 80; 

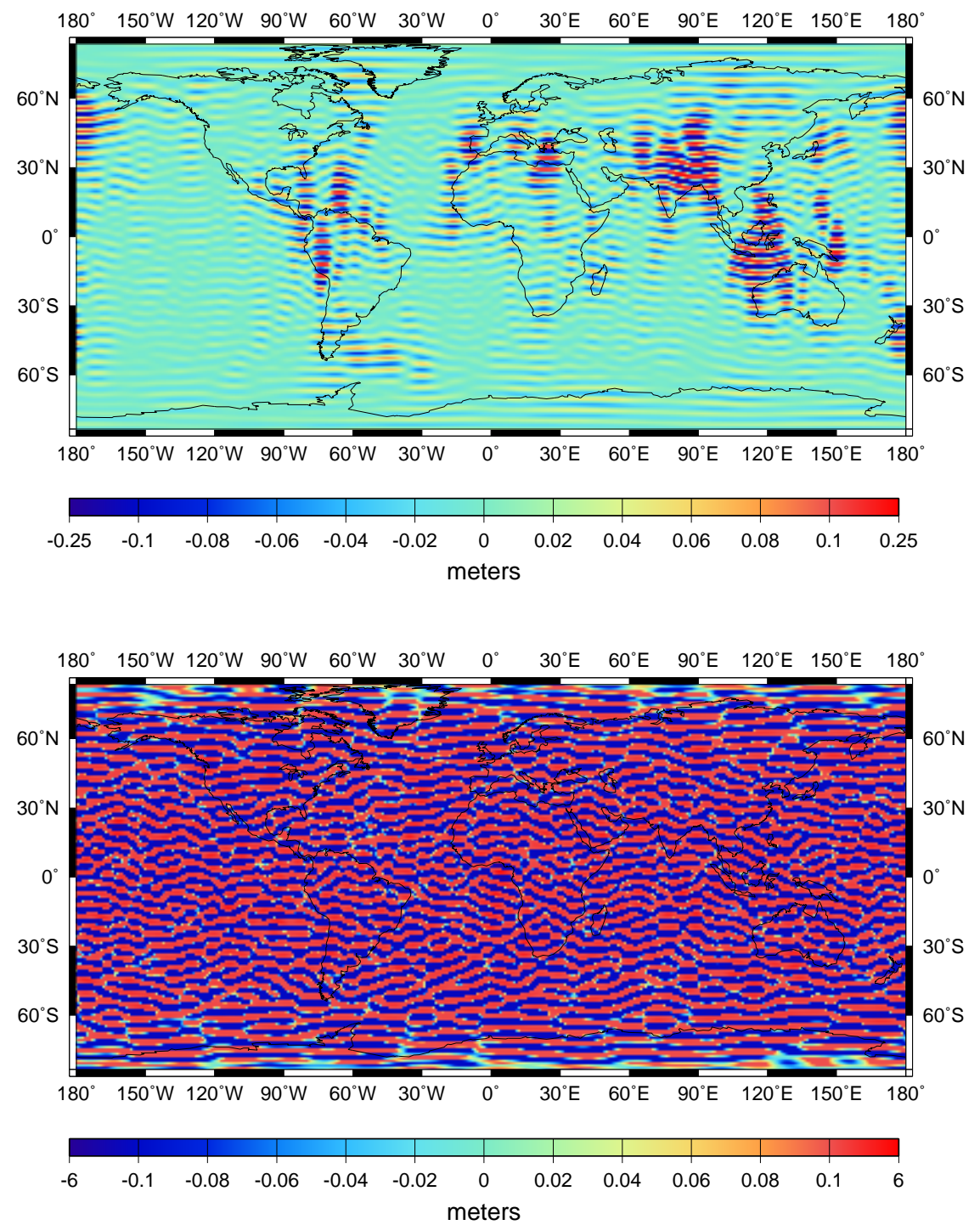

Fig. 1. Geoid height errors after the stand-alone inversion of noise-free SST data. The rms error is equal to $3.6 \mathrm{~cm}$; the maximum error reaches $24 \mathrm{~cm}$.
Fig. 2. Geoid height errors after the stand-alone inversion of noisy SST data without a regularization. The rms error is equal to $111 \mathrm{~cm}$; the maximum error reaches $534 \mathrm{~cm}$.
- Reference model of the Earth gravity field: JGM-3 (Tapley et al., 1996);

- Observation period: 10 days;

- Sampling rate: $15 \mathrm{sec}$;

_ "True orbit parameters: near-repeat, mean inclination $96.59^{\circ}$, elevation above the equator $246 \pm 7 \mathrm{~km}$.

In order to reduce the orbit discrepancies, the reference orbit was split into 10 one-day arcs, so that 10 unknown initial state vectors were included as additional parameters into the functional model. The maximum degree and order in the inversion was set to 80 , i.e. consistently with the "true model" definition.

First of all, noise-free satellite positions have been considered. The system of normal equations was built explicitly and solved by means of the Cholesky method; regularization was not applied. The obtained gravity field model is shown in terms of geoid height errors in Fig. 1. In order to quantify the quality of the model obtained, we have calculated the rms and the maximum (in the absolute sense) geoid height errors in the interval of latitudes $\pm 80^{\circ}$, so that the polar areas not cover by measurements are excluded. It turned out that the rms error is equal to $3.6 \mathrm{~cm}$, whereas the maximum error reaches $24 \mathrm{~cm}$. Thus, in spite of the absence of noise, the result of the inversion is not perfect. Most probably, the errors are caused by insufficiently small orbit discrepancies, so that the non-linearity of the problem cannot be fully ignored. In order to get rid of these errors, we should have applied an iterative improvement procedure. However, we leave a close investigation of these errors beyond the scope of our numerical study because they are much less than those caused by anticipated data inaccuracies.

To demonstrate the influence of data inaccuracies, an artificial quasi-random white noise of a modest amplitude, 1 $\mathrm{cm}$, was added to the satellite positions. Then, the data were inverted in the same way as in the previous case (i.e. without a regularization). The model obtained proved to be very erroneous (Fig. 2). In order to suppress the influence of 


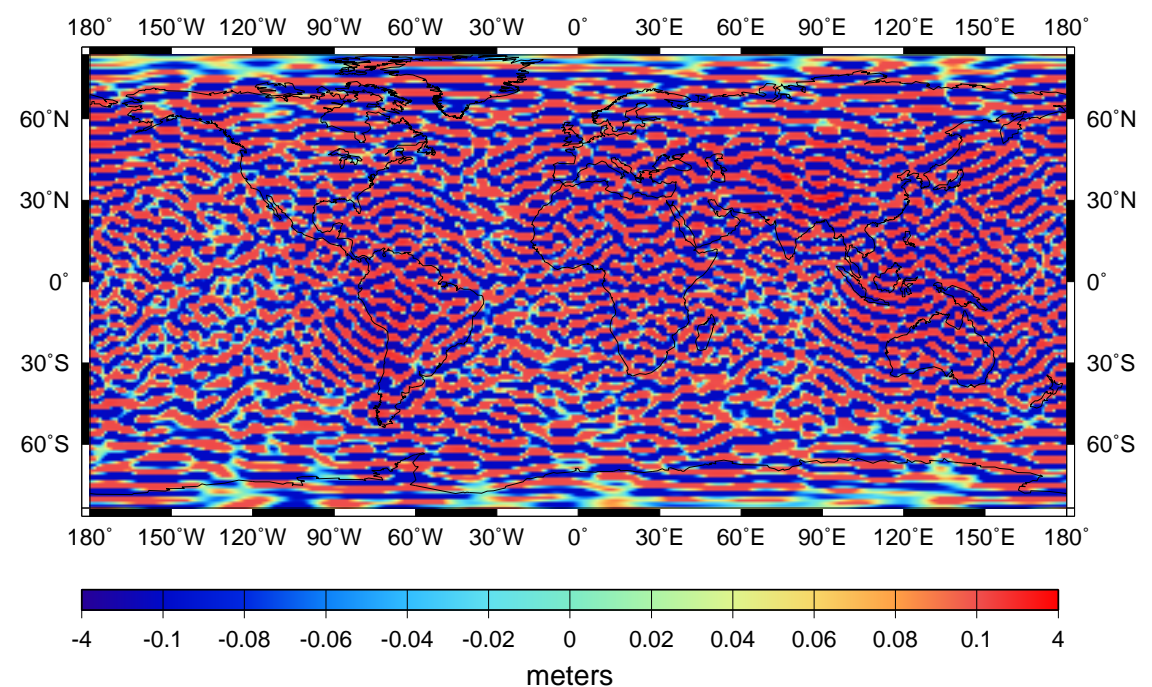

Fig. 3. Geoid height errors after the stand-alone inversion of noisy SST data with the optimal regularization. The rms error is equal to $46 \mathrm{~cm}$; the maximum error reaches $396 \mathrm{~cm}$.

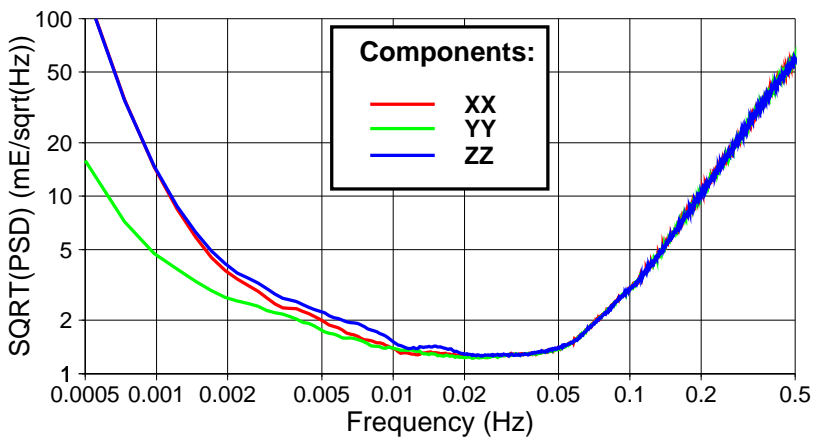

Fig. 4. Square-root of the noise PSD function used in the simulation of noisy SGG data

the noise, we have repeated the computations with the firstorder Tikhonov regularization applied (Tikhonov and Arsenin, 1977; Ditmar and Klees, 2002). But even the optimal selection of the regularization parameter allowed us to improve the model accuracy only about two times (Fig. 3).

\subsection{Stand-alone inversion of SGG data}

The second goal of our simulations was to demonstrate the influence of noise in SGG data, when these data are inverted in the stand-alone mode. In order to simulate a set of SGG data, we used the same 10-day "true" orbit that has been already presented above. The choice of the "true" and of the reference gravity field was also the same as in the previous examples. Other parameters of the simulation were as follows:

- Considered components of the SGG tensor: XX, YY, and ZZ;

- Sampling rate: $1 \mathrm{sec}$;

- Noise model: A realistic noise SQRT-PSD (square root of the power spectral density) from the report (ESA,
2000) (Fig. 4) was exploited to generate noise realizations.

The simulated SGG data were inverted into a model of the Earth's gravity field; as before, the maximum degree and order was set to 80. Regularization in this case was not applied; the system of normal equations was solved with the PCCG method. One can see that the model obtained this way is also characterized by significant inaccuracies (see Fig. 5).

\subsection{Joint inversion}

Finally, the above-presented sets of SST and SGG-data were inverted jointly. The normal equations were solved with (i) the Cholesky method based on the explicit building the normal matrix, and (ii) the PCCG method. As we expected, the PCCG method turned out to be much faster. On the SGI Origin 3800 super-computer with 30 processing elements, the computations according to the PCCG method took only 1.5 hours including 0.5 hour for input/output operations (mostly, reading the SST-related design matrix from external files). The explicit computation of the normal matrix and the inversion based on the Cholesky method took about 12 hours. The models obtained with both methods are identical; one of them is shown in Fig. 6. As expected, the joint inversion of SST and SGG data leads to a much higher accuracy than the stand-alone inversion of each data set.

\section{Discussion and conclusions}

The simulations performed allow one to understand better the power of a joint inversion of SST and SGG data. A standalone inversion of SST data cannot reproduce the Earth's gravity field accurately even in case of a modest truncation degree (namely, 80); this inference is also consistent with previous publications (e.g. Visser et al., 2001). The geoid height errors are characterized by a noticeable highfrequency pattern, where anomalies are extended in the East- 


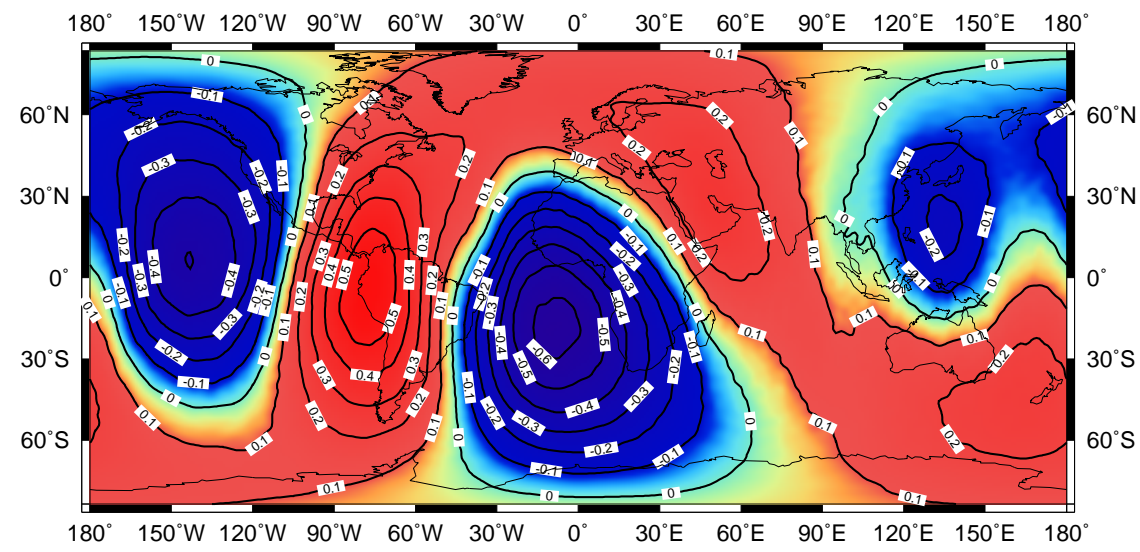

$\begin{array}{ccccccccccccc}-0.7 & -0.1 & -0.08 & -0.06 & -0.04 & -0.02 & \begin{array}{c}0 \\ \text { meters }\end{array} & 0.02 & 0.04 & 0.06 & 0.08 & 0.1 & 0.7\end{array}$

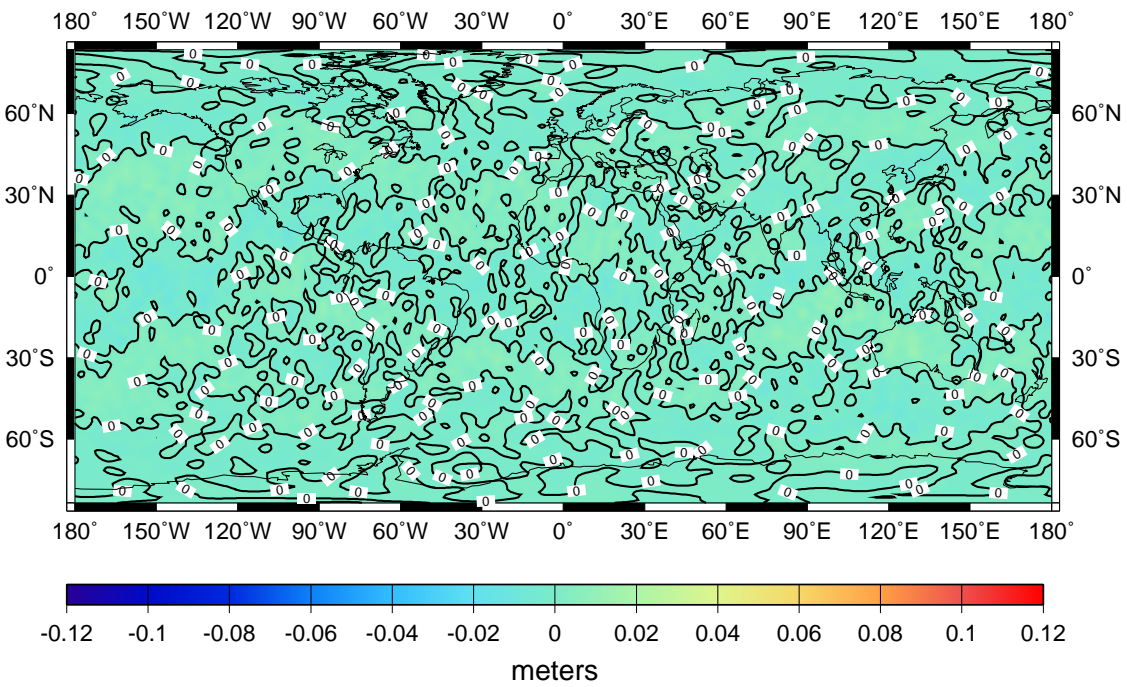

Fig. 5. Geoid height errors after the stand-alone inversion of noisy SGG data. The rms error is equal to $24 \mathrm{~cm}$; the maximum error reaches $64 \mathrm{~cm}$.
Fig. 6. Geoid height errors after the joint inversion of noisy SST and noisy SGG data. The rms error is equal to 0.3 $\mathrm{cm}$; the maximum error reaches $1.4 \mathrm{~cm}$.
West direction (Figs. 2, 3). This pattern can be explained by the fact that the Earth's gravity field, according to Newton's second law, manifests itself in satellite accelerations. In order to convert the accelerations into satellite positions, one should perform the double integration, which is a smoothing operation. Thus, by observing the satellite trajectory, we cannot percept fast along-track variations of the gravity field. Once the satellite orbit is polar, it is just these East-West variations which cause the observed error pattern.

SGG data alone lead to significant errors in the Earth's gravity field model, too. The behavior of the errors is, however, different: they show up, mostly, as a low-frequency pattern (Fig. 5). Such a behavior is dictated by the strong noise in SGG data at lowest frequencies (Fig. 4).

The joint inversion of SST and SGG data leads to results of much higher accuracy than any stand-alone inversion. This is because the joint inversion does not suffer from a lack of information like a stand-alone inversion: it takes the lowfrequency information from SST data and the medium-tohigh-frequency information from SGG data. What is cru- cial for a success of the joint inversion is a proper weighting of the data. Particularly, applying a proper filter to the SGG data in the course of inversion. Thanks to this filtering, the erroneous low-frequency contents in SGG data is downweighted. Thus, the result obtained is much better than the one which could be produced, e.g. by "mixing" results obtained with stand-alone inversion procedures.

It is worth noticing that we have been able to apply a proper weighting because we assumed that the stochastic properties of SGG and SST data are known. In practice, this may not be the case. Then, it might be necessary to apply a procedure for determination of the noise properties from the data themselves. (cf. Kusche, 2003).

Acknowledgements. We would like to express our special gratitude to Dr. E. Schrama for numerous valuable discussions related to our study. Besides, we would like to thank Dr. J. Kusche and two anonymous reviewers for a number of remarks and corrections that allowed us to improve the text of the article. In particular, the idea of a geometrical interpretation of the back-substitution at the level of the design matrix belongs to one of the reviewers. The research was 
financially supported by the Netherlands Space Research Organization SRON (grant eo-028). Computing resources were provided by Stichting Nationale Computerfaciliteiten NCF (grant SG-027). The GEODYN software was kindly provided by the Goddard Space Flight Center, Greenbelt, Maryland.

\section{References}

Bertsekas, D. P.: Constrained optimization and Lagrange multiplier methods, Academic Press, New York etc., 1982.

Colombo, O. L.: Notes on the mapping of the gravity field using satellite data, in Mathematical and Numerical Techniques in Physical Geodesy, Lecture Notes in Earth Sciences, edited by H. Sünkel, vol. 7, pp. 261-316, Springer, Berlin, 1986.

Ditmar, P. and Klees, R.: A Method to Compute the Earth's Gravity Field from SGG / SST data to be acquired by the GOCE Satellite, Delft University Press, 2002.

Ditmar, P., Klees, R., and Kostenko, F.: Fast and accurate computation of spherical harmonic coefficients from satellite gravity gradiometry data, Journal of Geodesy, 76, 690-705, 2003.

ESA: Gravity field and steady-state ocean circulation missions. Reports for mission selection. The four candidate Earth explorer core missions, SP-1233(1), European Space Agency, Noordwijk, 1999.

ESA: From Eötvös to mGal. Final report, ESA/ESTEC Contract 13392/98/NL/GD, European Space Agency, 2000.

Hestenes, M. R. and Stiefel, E.: Methods of conjugate gradients for solving linear systems, Journal of Research of the National Bureau of Standards, 49, 409-436, 1952.

Jekeli, C.: The determination of gravitational potential differences from satellite-to-satellite tracking, Celestial Mechanics and Dynamical Astronomy, 75, 85-101, 1999.

Klees, R., Ditmar, P., and Broersen, P.: How to handle colored observation noise in large-scale least-squares problems, Journal of
Geodesy, 76, 629-640, 2003.

Kusche, J.: A Monte-Carlo Technique for Weight Estimation in Satellite Geodesy, Journal of Geodesy, 76, 641-652, 2003.

Lemoine, F. G., Kenyon, S. C., Factor, J. K., Trimmer, R. G., Pavlis, N. K., Chinn, D. S., Cox, C. M., Klosko, S. M., Luthcke, S. B., Torrence, M. H., Wang, Y. M., Williamson, R. G., Pavlis, E. C., Rapp, R. H., and Olson, T. R.: The development of the joint NASA GSFC and the National Imagery and Mapping Agency (NIMA) geopotential model EGM96. NASA/TP-1998-206861, NASA GSFC, Greenbelt, Maryland, 1998.

Rowlands, D., Marshall, J. A., McCarthy, J., Moore, D., Pavlis, D., Rowton, S., Luthcke, S., and Tsaoussi, L.: GEODYN II system description, vol. 1-5, Contractor report, Hughes STX Corp., Greenbelt, MD, 1995.

Schäfer, C.: Space Gravity Spectroscopy, Deutsche Geodätische Kommission, Reihe C, Heft Nr. 534, München, 2001.

Schuh, W. D.: Tailored numerical solution strategies for the global determination of the Earth's gravity field, Mitteilungen der geodätischen Institute der Technischen Universität Graz. Folge 81. Graz, 1996.

Tapley, B. D., Watkins, M. M., Reis, J. C., Davis, G. W., Eanes, R. J., Poole, S. R., Rim, H. G., Schutz, B. E., Shum, C. K., Nerem, R. S., Lerch, F. J., Marshall, J. A., Ulosko, S. M., Pavlis, N. K., and Williamson, R. G.: The joint gravity model 3, J. Geophys. Res, B, 101, 28,029 - 28,049, 1996.

Tikhonov, A. N. and Arsenin, V. Y.: Solutions of ill-posed problems, V.H.Winston and Sons, Washington, D.C., 1977.

Tscherning, C. C.: Personal communication, 2002.

Visser, P. N. A. M., van den IJssel, J., Koop, R., and Klees, R.: Exploring gravity field determination from orbit perturbations of the European Gravity Mission GOCE, Journal of Geodesy, 75, 89-98, 2001.

Wolff, M.: Direct measurements of the Earth's gravitational potential using a satellite pair, Journal of Geophysical Research, 74, 5295-5300, 1969. 